\title{
Une recherche-action: Connaître, accompagner et provoquer le changement en sciences de l'éducation
}

\section{Véronique Pelt et Débora Poncelet}

Cet article a pour visée la présentation de la théorie sous-jacente à la recherche-action appliquée aux sciences de l'éducation. La revue de la littérature a permis de suivre son évolution depuis les années 1940 et de proposer une définition basée sur les critères épistémologiques qui la caractérisent afin de mettre en exergue les principes nécessaires à son agir. Un tableau comparatif permet de distinguer la recherche-action de la recherche expérimentale.

Le présent article a pour visée de présenter la théorie sous-jacente à la rechercheaction (RA) appliquée aux sciences de l'éducation dans le cadre éducatif. Il existe en effet «théoriquement et pratiquement, diverses manières de relier la recherche à l'action" (Goyette \& Lessard-Hébert, 1987, p. 13). Il semble alors fondamental d'exposer son élaboration conceptuelle, afin de théoriser, grâce à cet éclairage, sa mise en œuvre, pour éviter d'entrer dans l'action en oubliant de prendre en considération la recherche. En effet, «the approach is only action research when it is collaborative, though it is important to realise that the action research of the group is achieved through the critically examined action of individual group members» (Kemmis \& McTaggart, 1988, p. 5). La question de fond que doit se poser tout chercheur sur son objet d'étude avant de s'engager est de savoir quels en sont les éléments indispensables et constitutifs. Il en est de même pour une RA réalisée dans un cadre éducatif.

Le choix de ce champ est inhérent à la place accordée par les sciences de l'éducation notamment dans le domaine de la relation entre l'école et la famille. En effet, depuis un peu moins d'un demi-siècle, elles l'ont fortement investi. Ce partenariat fait l'objet d'une préoccupation qui ne s'est jamais démentie. Les écrits sur ce sujet ne se comptent plus, tant chaque année apporte son lot de réflexions théoriques (Bouchard, 1998; Bouchard \& Kalubi, 2006; Epstein, 1996, 2001; Hoover-Dempsey et al., 2001; Hoover-Dempsey \& Sandler, 1995, 2005), d'études de validations (Deslandes \& Bertrand, 2005; Deslandes, Potvin \& Leclerc, 1999; Green, Walker, Hoover-Dempsey \& Sandler, 2007), de recherches empiriques (Ferrara, 2009; Georgiou \& Tourva, 2007) ou encore de méta-analyses (Hill \& Tyson, 2009; Hohlfeld, Ritzhaupt \& Barron, 2010). 
La littérature de recherche (Epstein, 1996) a mis en évidence les bénéfices que l'enfant pouvait retirer d'une telle collaboration. Il semble en effet que les retombées se fassent sentir tant au niveau des résultats scolaires que des comportements et des attitudes à l'école. Selon Hoover-Dempsey, Walker, Jones et Reed (2002), l'engagement des parents dans l'éducation de leur enfant est accru lorsque les parents se rendent compte que les enseignants les tiennent informés, accordent de la valeur à leurs interventions éducatives et leur fournissent des conseils spécifiques sur l'aide à apporter aux apprentissages réalisés par l'enfant. Lorsque les parents s'investissent de façon efficace dans l'éducation de leur enfant, ils prennent également davantage conscience de l'impact que peut avoir leur comportement sur la réussite scolaire de ce dernier. De plus, lorsque les parents perçoivent que l'école accueille de façon favorable leur engagement, ils ont tendance à participer encore plus activement et se montrent satisfaits de la qualité des enseignements dont bénéficie leur enfant. En retour, la satisfaction des parents a des répercussions positives sur les enseignants. Une communication efficace entre l'école et les familles renforce donc le lien entre les attentes relatives aux apprentissages que développent les deux environnements éducatifs et clarifie le rôle que chacun des partenaires peut apporter au succès scolaire de l'enfant.

Toutefois, lorsqu'un chercheur envisage un changement comportemental ou sociétal de ce type, ses moyens d'actions sont relativement limités. Les sciences humaines en général et de l'éducation en particulier ont alors privilégié la RA comme outil de réflexion et de praxéologie, car sa mise en place semblait être à même de répondre à leurs attentes. Elle s'est ainsi largement développée, au Québec et dans les pays anglo-saxons, dès la fin des années 1970. Nous avons ainsi pu constater que la RA est actuellement l'une des modalités de recherche les plus utilisées dans les études empiriques visant la formation des pédagogues concernant une modification comportementale ou relationnelle (Brighton \& Moon, 2007; Desgagné, Bednarz, Lebuis, Poirier \& Couture, 2005; Perrett, 2003; Tisseron, 2008). Parallèlement, de nombreux manuels sont publiés relatant tant la méthodologie appliquée que des recherches empiriques. Ainsi, dans son ouvrage "Action Research in Workplace Education», Taylor (2002) ne se contente pas de mettre en évidence une méthodologie en sept étapes, mais évoque leur mise en œuvre au travers de huit études ainsi que six exercices permettant de mieux définir son objet de recherche.

Fondamentale dans les sciences sociales, cette approche responsabilise le chercheur dans la recherche mais aussi et surtout au sein même de la communauté qu'il co-analyse et où il intervient comme co-auteur du changement.

Divisé en quatre parties, cet article apporte un éclairage, dans un premier temps, sur l'histoire de l'évolution de la RA, puis se propose, dans un deuxième temps, de la définir et évoque, dans un troisième temps, ses principes d'action. 


\section{Une recherche en évolution}

La RA telle que nous la connaissons aujourd'hui est le produit d'une évolution de presque sept décennies.

Ses origines remontent, selon Thirion (1980, cité par Goyette \& LessardHébert, 1987), au mouvement de l'École nouvelle inspirée par Dewey après la Première Guerre Mondiale. Il postulait l'importance de lier la pensée à l'action et avait pour objectif la création d'une science de l'éducation où enseignants et élèves auraient un rôle à jouer dans la construction des savoirs issus de la praxis. Selon Poncelet et Voz (2004), cette nouvelle conception de la recherche est la résultante de deux constats:

- Les recherches en laboratoire sur les pratiques enseignantes sont décevantes et n'ont que peu d'impact (sentiment d'échec);

- Les chercheurs de l'époque sont à la recherche d'un idéal démocratique de travail coopératif et communautaire. Le milieu du $20^{\mathrm{e}}$ siècle (d'aprèsguerres) se caractérise par une volonté de travailler ensemble, d'échanger des idées et des connaissances. Shumsky (1956) le traduit ainsi: «A way of creating a social acting where people can work together, dream together of a better community».

Citons également comme précurseur Collier (1945) qui enracinait ses recherches au terrain et reliait l'action à la recherche (Dolbec \& Prud'Homme, 2009). Enfin, pour Coenen (2001), la paternité de cette nouvelle démarche scientifique reviendrait à Paulo Freire. Avec lui, ce sont les dimensions de participation et d'émancipation qui entrent dans la recherche; les acteurs y sont perçus comme compétents et aptes à gérer eux-mêmes les actions et à transformer leur propre réalité. Les notions de confiance mutuelle, de dialogue ouvert entre chercheurs et protagonistes, de niveau d'égalité dans les connaissances de chacun, y prennent tout leur sens. Depuis les années 1940, divers mouvements se sont succédés pour donner naissance à la RA telle que pratiquée de nos jours.

La première génération (terme emprunté à King \& Lonnquist, 1994) de RA, dite positiviste (Anadón \& Savoie-Zajc, 2007) ou de consensus orthodoxe (Coenen, 2001) débute avec les travaux de Lewin dans le champ psychosocial. Généralement associé à ce concept, ce chercheur a réussi, dans les années 1940, à ériger la RA comme orientation scientifique dans le domaine de l'intervention psychosociale et a été le premier à employer le terme de "Action research" proprement dit. Appliquant une démarche scientifique rigoureuse issue de la psychologie, il a introduit les notions de changement social et de dynamique de groupe. Chercheur avant tout, il explore le concept de changement, en théorise les étapes qu'il valide dans l'action. Il a ainsi pu constater que le changement était d'autant plus important s'il s'adressait au groupe plutôt qu'à l'individu. Sa vision l'amène à privilégier les problèmes sociaux réels comme objet d'étude et à inclure dans sa réflexion les protagonistes qui y sont liés. L'action s'imbrique alors à la recherche et amène le chercheur à aller sur le terrain et à quitter son laboratoire 
où il était jusqu'alors isolé pour s'allier aux parties prenantes dans l'analyse de la situation problématique et sa résolution dans l'action. Il met ainsi en place une approche globalisante intégrant recherche, action et formation. Cependant, le chercheur y maintient son rôle objectif et neutre, il reste le détenteur du savoir où les acteurs sociaux ne sont qu'une source passive d'informations. Le consensus orthodoxe prime toujours: la méthodologie de la recherche sociale est identique à celle des sciences de la nature; le chercheur est le plus à même d'analyser les comportements individuels (Coenen, 2001).

Pour résumer et en se basant sur la classification de Zuber-Skerritt (1992, cité dans Savoie-Zajc, 2001), nous pouvons dire que cette génération est caractérisée par une position épistémologique de type technique: le changement est la résultante de la mise en place de différentes procédures, le chercheur y occupe une place centrale dans l'accompagnement mais est externe à la situation (objectivité). Le paradigme associé est positiviste.

Précisons enfin que Corey (1953, cité dans Goyette \& Lessard-Hébert, 1987) a étendu cette méthode aux secteurs éducatifs américains. Pour cet auteur, la recherche était synonyme de changement si elle se faisait de concert avec les praticiens qui devaient réfléchir sur les moyens d'améliorer leurs pratiques. Il fut l'un des premiers à présenter des résultats (Dolbec \& Prud'Homme, 2009).

La deuxième vague apparaît dans les années 1970, où elle constitue un paradigme indépendant dans les sciences sociales. L'épistémologie y est fonctionnaliste. Son émergence est due à la combinaison de plusieurs phénomènes:

- D'abord la remise en cause dans les sciences sociales du paradigme positiviste permettant alors l'apparition d'une théorisation qui leur est propre et qui favorise l'évolution de la RA;

- Ensuite, l'absence d'informations sur le déroulement de la recherche ellemême, ne permettant pas une remise en question de l'intervention intraaction;

- S’y ajoute enfin l'interdisciplinarité, l'inclusion en place de l'exclusion (recherche et actions), la prise en compte des relations avec l'environnement permettant d'envisager la recherche de manière différente et agissant directement sur le rôle du chercheur et sur sa possible implication dans la recherche où les savoirs et les compétences des acteurs sont tout autant source de bénéfice dans la compréhension d'un phénomène que ceux du chercheur.

Les travaux de Stenhouse (1975), Elliott (1977) et Schön (1987) sont associés, selon Dolbec et Prud'Homme (2009), à cette vague avec une centration sur les besoins des praticiens, ainsi qu'une participation active des acteurs adoptant le rôle de co-chercheur. Stenhouse préconisait que «curriculum research and development ought to belong to the teacher» (1975, p. 142). Il va plus loin en affirmant «it is not enough that teachers' work should be studied: they need to study it themselves» (p. 143). Il introduit ainsi le concept d'enseignant-chercheur, slogan de cette nouvelle génération (Lapassade, 1993). La RA adopte alors une visée collaborative et évolutive (Giust-Desprairies, 2001). Kolb apparaît également 
comme pilier de cette deuxième génération grâce à son modèle d'apprentissage expérientiel (1984) qui indique que chaque individu est à même de créer la connaissance:

- sur la base de leur expérience concrète,

- par l'observation et la réflexion sur leur expérience,

- en formant des concepts abstraits et des généralisations,

- et en testant l'implication de ces concepts dans de nouvelles situations, ce qui conduit à une nouvelle expérience concrète et par conséquent, à l'amorce d'un nouveau cycle (Dolbec \& Prud'Homme, 2009, p. 539).

Les deux grandes figures de cette génération ont donné un nouveau souffle à la RA en octroyant une place prépondérante à l'enseignant en tant que chercheur (Stenhouse, 1975) et au processus d'apprentissage expérientiel (Kolb, 1984) mettant ainsi en évidence un système circulaire basée sur l'expérience pratique (ou action concrète), l'observation réflexive, la conceptualisation abstraite et l'expérimentation active. Cette RA est selon Zuber-Skerritt (1992, cité dans SavoieZajc, 2001) pratique, c'est-à-dire sous-tendu par le paradigme interprétatif. Le changement est l'aboutissement d'une réflexion sur la praxis et permet un développement de ses compétences. Les intervenants occupent pleinement une place de co-chercheur.

La troisième génération de RA est marquée par un élargissement du concept et par un paradigme critique. Avec Carr et Kemmis (1986), c'est la notion émancipatrice qui se lie dorénavant aux concepts de 'Recherche', 'Action' et 'Participation'. Le changement dans les pratiques éducatives ne peut se mettre en place que si les enseignants adoptent eux-mêmes un regard critique envers leur pratique et l'environnement dans lequel elle s'effectue. Alors les co-chercheurs ne se contenteront plus de modifier leur praxis en tant que centre du processus de recherche mais également le contexte sociopolitique dans lequel ils évoluent. Ces chercheurs font notamment référence à "the accountability movement» qui a "galvanisé et politisé les praticiens» (Lapassade, 1993, p. 5). L'école, devenant cible de critiques, se devait de rendre des comptes à la société, devait gérer la crise institutionnelle qui l'envahissait. Carr et Kemmis proposent donc une démocratisation de la recherche elle-même faite de "praticiens à partir de leur propre pratique. [...] La démocratie n'est plus dans la conduite dite 'démocratique' des petits groupes, elle passe dans la démocratisation de la recherche (action) elle-même, c'est-à-dire de la production d'un savoir qui n'est plus un savoir d'expert» (Lapassade, 1993, p. 5). Carr et Kemmis décident de «sauter dans l'action et de considérer leur recherche comme une sorte d'intervention sociale pour changer le système» (Dolbec \& Prud'Homme, 2009, p. 541). Leur vision de la recherche prend en considération les enjeux du terrain. Elle ne s'arrête plus au développement personnel et professionnel des enseignants mais intègre des éléments de tout le système. Il ne s'agit plus de modifier la praxis mais bien de l'étudier.

Anadón et Savoie-Zajc (2007) et Lapassade (1993) reprennent les cinq caractéristiques de la RA critique énoncées par Carr et Kemmis (1986): 
- Rejet des notions positivistes de rationalité, d'objectivité et de vérité.

- Posture réflexive permettant la prise de conscience des postulats et des valeurs sous-jacents à l'acte professionnel.

- Recadrage et mise à distance permettant la prise de conscience du biais possible dans la clarification des valeurs et postulats sous-jacents à la pratique.

- Ouverture à reconnaître la valeur des choix.

- Lien clair entre recherche et pratique, l'une guidant l'autre.

La RA apparaît, au sein de cette troisième vague, comme objet de conscientisation des masses dans un but de modification systémique et devient, dès lors, fortement politique. Ainsi, dans des RA issues d'Amérique du Sud (Paulo Freire) ou d'Inde (Rahje Tandon), l'objectif recherché des RA menées est bien une appropriation des savoirs et des moyens pour s'autogérer afin de s'opposer au pouvoir politique et de générer des changements sociaux (Doblec \& Prud'Homme, 2009). À ce titre, elle peut se voir dotée du paradigme de l'humanisme radical tel que décrit par Burell et Morgan (Dolbec, 2003). Afin de classifier les différentes théories sociales, ces auteurs (1979) ont été les premiers à utiliser la notion de paradigmes (Rouleau, 2007) et en ont définis quatre de type sociologique: fonctionnaliste, interprétatif, radical humaniste et radical structuraliste. Si la RA appartient au paradigme radical humaniste, c'est bien parce qu'elle met en évidence, lors de cette troisième génération, la domination, qu'elle la critique et tente de provoquer un changement par le dialogue. Les termes de conflit avec le pouvoir, de confrontation ou de revendication y prédominent. La RA est dite émancipatrice (Zuber-Skerritt, 1992, cité dans Savoie-Zajc, 2001). Elle reprend les mêmes volontés de changement et d'amélioration des pratiques mais ne s'arrête pas au niveau individuel et veut atteindre un changement du système luimême. Le paradigme sous-jacent est la théorie critique ainsi que les travaux d'Habermas concernant sa "theory of knowledge-constitutive interests» et servant de base à la réflexion de Carr et Kemmis.

Enfin, une quatrième génération aurait fait son apparition. Elle poursuivrait des buts émancipateurs et serait menée essentiellement par des groupes afin d'améliorer la pratique éducative et plus généralement l'éducation (Savoie-Zajc, 2001).

"Actuellement, les pratiques de la RA ont tendance à réduire l'aspect politique du projet pour devenir des outils d'intervention andragogiques et psychopédagogiques: elles visent moins des transformations des structures externes que des adaptations des structures internes» (Van der Maren, 2003, p. 99). Lors de la précédente génération, les co-chercheurs rejetaient leur neutralité dans l'appréhension du monde social au profit d'une conception propre à chacun impliqué dans l'action. Ils avaient pour objectif non seulement d'améliorer leur praxis mais aussi la société environnante. En devenant moins politique dans cette quatrième génération, la RA se recentre sur l'étude de la praxis avec une triple finalité d'action, de formation et de recherche. Toutefois, elle reste encore, selon Stringer (1999), la manière la plus appropriée pour responsabiliser les chercheurs au sein de leur communauté et les engager dans un processus démocratique. Elle 
serait ainsi actuellement assimilée à la RA communautaire de Stringer (1999, cité dans Champagne, 2007) et appartiendrait au courant interprétatif où les procédures consensuelles et participatives amènent les acteurs à poser un regard critique et systématique sur leur problème, à analyser de façon approfondie leur situation et à trouver des solutions appropriées. Trois adjectifs la définissent et en sont indissociables: démocratique, équitable et libératrice (Stringer, 1999, p. 10).

Pour résumer, la structure de la RA s'est modifiée selon quatre phases: de positiviste en première génération (années 1940-50: Lewin et Corey), elle devient pragmatique et participative (1970-80: Stenhouse et Kolb), puis émancipatrice et critique (années 1980-90: Carr \& Kemmis, Kemmis \& McTaggart), pour aboutir en quatrième génération à une recherche plus communautaire et à visée andragogique (Stringer, depuis les années 1990). La figure suivante reprend ces divers éléments. Chacun des niveaux de cette pyramide se veut intégrateur des éléments qui le précèdent: on peut réellement parler d'une évolution de la RA marquée par une volonté intégratrice plutôt que renonciatrice de certaines des composantes de la vague précédente. Chacune des périodes a su faire siennes les caractéristiques issues de l'autre mouvement tout en y apportant un élément novateur et amplificateur.

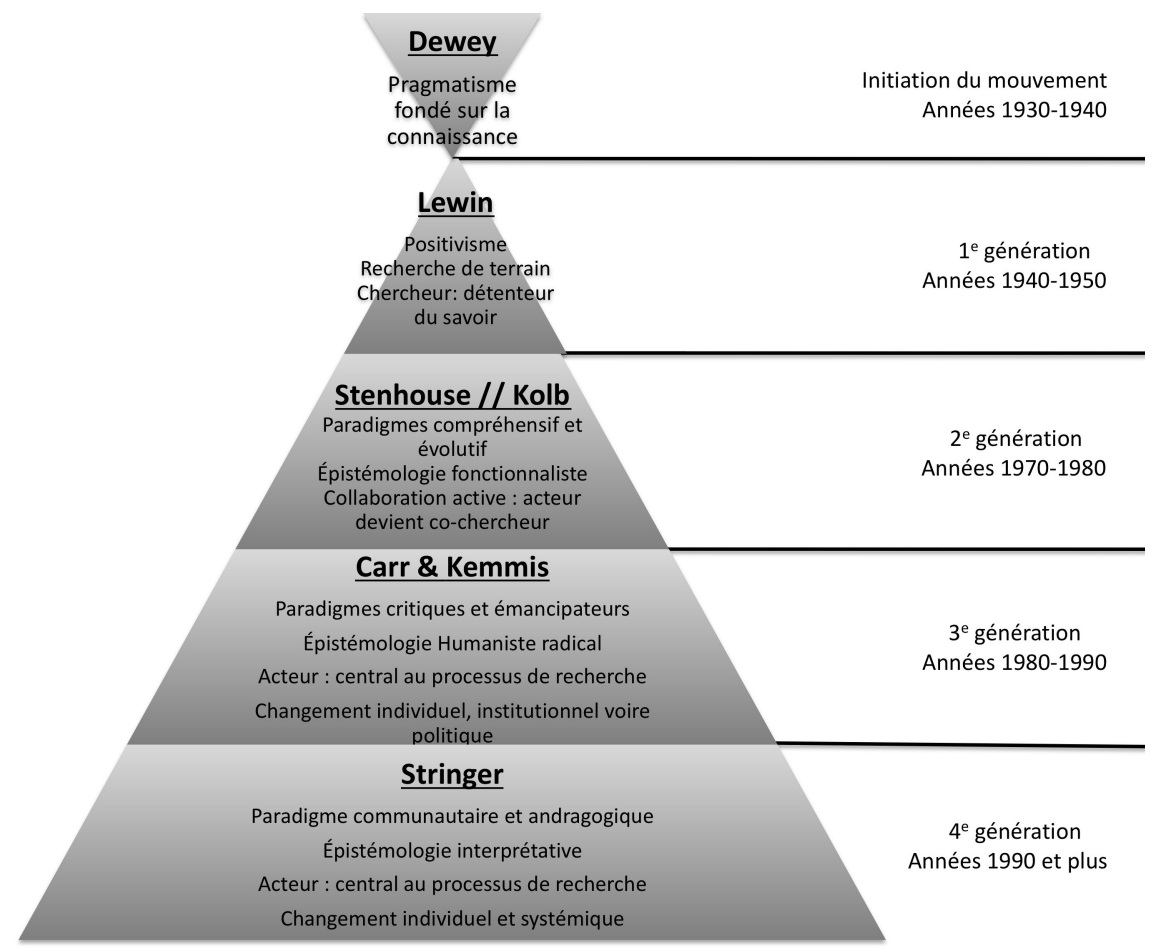

Figure 1. Quatre générations de recherche-action 
Pour finaliser cette présentation, nous pouvons arguer comme beaucoup d'auteurs l'ont souligné (Anadón \& Savoie-Zajc, 2007; Dolbec, 2003; Dolbec \& Prud'Homme, 2009; Goyette \& Lessard-Hébert, 1987; Lapassade, 1993) que la RA se caractérise selon trois dimensions:

- Les finalités attribuées à la RA contribuent en outre à sa définition et à l'orientation méthodologique qui en suivra. Selon Goyette et Lessard-Hébert (1987), il existe quatre fonctions possibles: 1) une construction de la connaissance, 2) un rôle critique l'opposant à la science traditionnelle, 3) un changement social et 4) une fonction de formation. Celles-ci se situent à trois niveaux, celui de la recherche, de l'action et de la formation.

- Les fondements idéologiques ou épistémologiques.

- Les instrumentations (approches/méthodes/méthodologies et instruments/outils).

En cela, Goyette et Lessard-Hébert (1987) rejoignent Paillé (1994), pour qui «la méthodologie de recherche scientifique est décisive quant à l'adéquation, à la finesse et l'impact d'une recherche» (p. 215). Toutefois, ces auteurs soulignent l'absence de méthodologie correspondant à une RA unique, mais octroie à chaque RA une souplesse méthodologique qui attend des chercheurs une adaptation à l'objet de recherche. «La méthodologie de la recherche-action part du milieu pour retourner au milieu concerné» (Tremblay \& Bonnelli, 2007, p. 67). Le fonctionnement méthodologique d'une RA peut être considéré, à l'instar de Lavoie, Marquis et Laurin (2003, cité dans Tremblay \& Bonnelli, 2007, p. 67) "comme une spirale dont chaque cycle comprend différentes phases: réflexion initiale, précision du problème et de son contexte, planification de l'action, action et observation, évaluation et prise de décisions».

Partant de son évolution, nous sommes maintenant plus à même d'aborder ce concept du point de vue de sa définition.

\section{Vers une redéfinition de la recherche-action}

Compte tenu de sa nature polysémique, il existe une pléthore de définitions qui proposent finalement une description plutôt qu'une définition littérale figée (Poncelet \& Voz, 2004). Définie comme orientation scientifique par Lewin en 1946, cette méthode permet, une "meilleure adéquation de la recherche empirique à la complexité du champ éducatif» (Paillé, 1994, p. 215). Stratégie de recherche et de formation dans un premier temps, elle devient au fil du temps stratégie du changement social. Nous pouvons souligner quelques définitions pertinentes:

- «Démarche de compréhension et d'explication de la praxis des groupes sociaux, par l'implication des groupes eux-mêmes, dans le but d'améliorer leur praxis» (Cardinal \& Morin, 1993. p. 2), 
- «Action délibérée, visant un changement dans le monde réel, engagée sur une échelle restreinte, englobée par un projet plus général et se soumettant à certaines disciplines pour obtenir des effets de connaissance ou/et de sens» (Dubost, 1988. pp. 45-46),

- "Système d'activités humaines qui vise à faire émerger un processus collaboratif dans le but de produire un changement dans le monde réel» (Dolbec, 2003, p. 527).

Ces définitions restent toutefois parcellaires. On y note l'absence de deux des dimensions de la RA: l'aspect de recherche (développement de théories sur le changement) et l'aspect de collaboration.

En ce qui nous concerne, à l'instar de Tremblay et Bonnelli (2007, p. 66), nous pensons qu'une RA se doit d'être co-construite, qu'elle demande de faire participer les acteurs sociaux à un processus, dans un but de production de connaissances théoriques et pratiques, de diagnostic de la situation, de propositions d'actions et de leur réalisation. En nous basant sur le descriptif des enjeux de la recherche appliquée en pédagogie (Van der Maren, 2003), nous pouvons ajouter qu'une RA réunit ces caractéristiques:

- «Pragmatique» (pp. 25-26), elle vise la résolution de problème de dysfonctionnement.

- «Ontogénique» (pp. 28-29), elle permet à ses protagonistes un développement personnel et un perfectionnement grâce à une réflexion sur l'action;

- «Politique» (p. 26), elle a pour but le changement des pratiques individuelles et institutionnelles.

- «Nomothétique» (pp. 23-24) elle ambitionne une production de savoir par un travail de méta-recherche.

La notion de connaissance y est un élément central, car elle est tout à la fois objet d'étude et moteur de l'action. Une des finalités de la RA pouvant être également l'appréhension des représentations des participants sur un thème, la connaissance permettra alors l'organisation de ces représentations. De la dualité de cette imbrication, nous constatons que «tout progrès de l'action profite à la connaissance et tout progrès de la connaissance profite à l'action» (Morin, 1980, cité dans Goyette \& Lessard-Hébert, 1987).

Dans ce cadre de recherche, le temps joue également un rôle particulier. Il est en effet un élément constitutif de la structure méthodologique. S'il est important dans toute étude de définir l'espace-temps dans lequel elle s'inscrit, au sein d'une RA, l'aspect temporel y occupe une place à part, puisqu'il est inhérent à la recherche elle-même: «Le temps modifiera et la réalité observée et le produit de [la] réflexion [des chercheurs]» (Goyette \& Lessard-Hébert, 1987, p. 1). Le temps doit intervenir comme outil de réflexion et d'évaluation de l'action. Force est de constater avec Paillé (2007, p. 149) que la logique de ce type de recherche est "presque toujours progressive, itérative et récursive» et seul le temps peut permettre ces progressions, itérations et récursivités. 
Enfin, le groupe sert à la méthodologie. Comme le temps et la connaissance, il est partie prenante dans la structure méthodologique. Il devra en outre, comme le souligne Lewin, être de taille restreinte pour une plus grande efficacité surtout si la RA a pour but la transformation d'attitudes et de comportements dans le secteur de l'activité sociale (approche «micro» des processus).

Ces différents éléments nous permettent maintenant de procéder à un comparatif, sous forme de tableau (tableau 1), entre recherche-action et recherche expérimentale (Bazin, 2006; Berthon, 2005; Savoie-Zajc, 2001; Van der Maren, 2003) qui achèvera cette tentative de définition.

\section{Principes d'action}

Une RA, comme toute recherche quelle qu'elle soit, doit satisfaire aux principes d'actions tels qu'énoncés par Poncelet et Voz (2004, p. 161), reprenant les points de vue déontologique de de Landsheere (1976):

- La recherche doit se faire avec l'accord de toutes les parties concernées.

- La recherche ne peut ni nuire à la santé physique ou mentale des sujets, ni les handicaper, de quelque façon que ce soit dans leur apprentissages.

- La recherche ne peut violer ni l'intimité de l'individu ni celle des familles (p. 375).

Par ailleurs, elle satisfait à des principes d'action pratique qui lui sont propres (Mouvet, Munten \& Jardon, 2001), notamment dans le cadre déterminé de l'école:

- Établir les propositions d'action en commun avec les participants de la recherche.

- Ancrer les pratiques nouvelles dans les pratiques antérieures positives.

- Rendre les enseignants autonomes dans la réalisation et la gestion de l'action innovante afin d'assurer la pérennité au sein de l'établissement au-delà de notre intervention et présence sur le terrain.

- Demeurer critiques quant aux impacts des propositions émises, qui tirées de leur contexte d'origine, peuvent s'avérer plus néfastes que bénéfiques.

- Tenir compte des ressources humaines, financières et autres, ainsi que des contingences propres à chaque entité lors de l'élaboration du projet, des actions à mettre en œuvre, leur implantation et leur évaluation (pp. 6-8).

Outre ces principes, Mouvet (2009) précise un certain nombre de conditions favorables et nécessaires permettant l'atteinte des objectifs relationnels d'une RA «fondés sur l'éthique de la relation à l'autre qui se veut de l'ordre de l'écoute plutôt que du regard» (p. 70). Dix principes sont ainsi énoncés comme propices à la construction collective. Ils ont été formalisés par ce que Mouvet nomme le "groupe porteur» (p. 23) lors de la mise en place de réseaux porteurs d'innovations scolaires. Leur importance étant identique, ils sont présentés par ordre alphabétique: «accueillir, clarifier (reclarifier-cerner-circonscrire), co-construire 
Tableau 1. Comparaison entre recherche-Action et Recherche Expérimentale

\begin{tabular}{|c|c|c|c|c|c|c|c|c|c|c|c|}
\hline 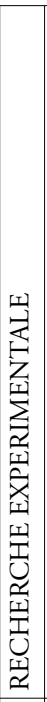 & 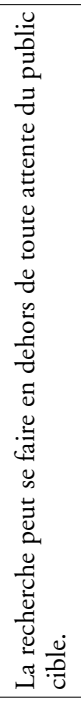 & 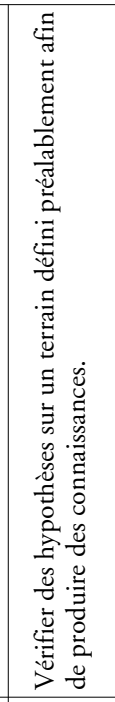 & 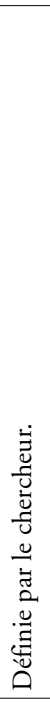 & 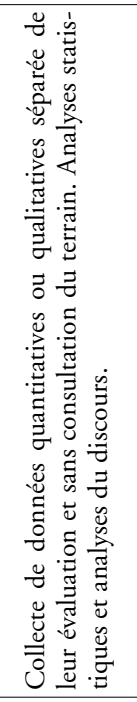 & 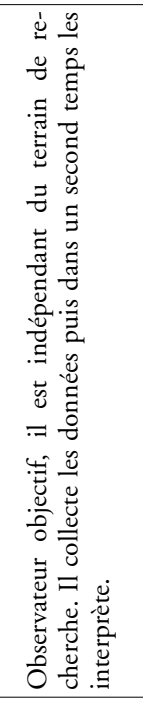 & 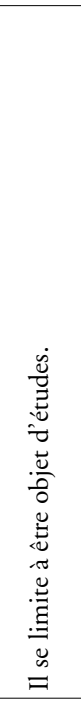 & 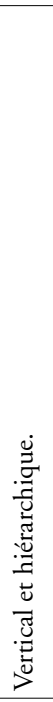 & 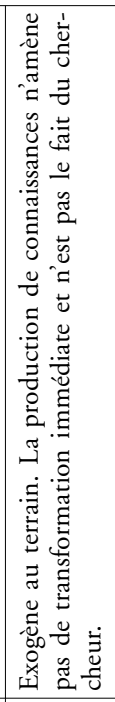 & 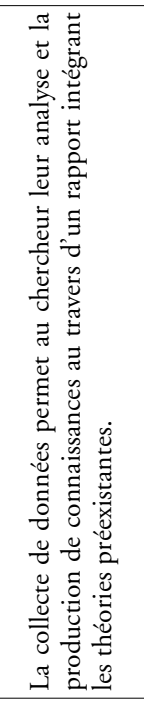 & 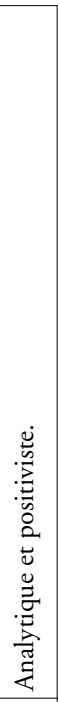 & 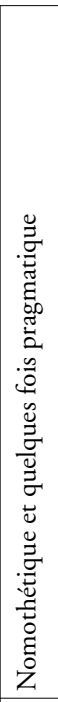 \\
\hline 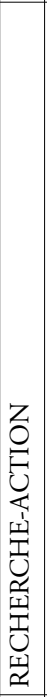 & 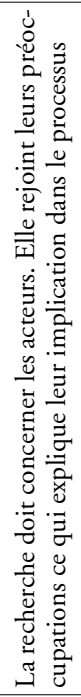 & 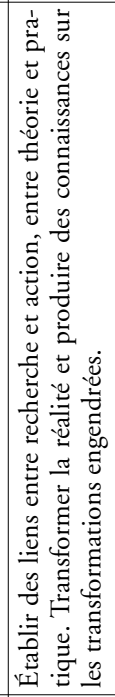 & 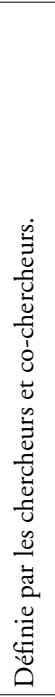 & 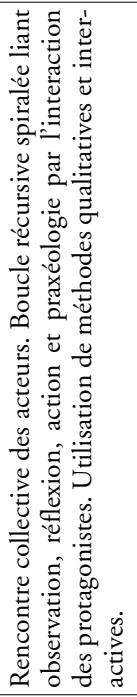 & 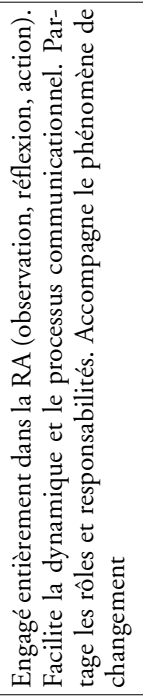 & 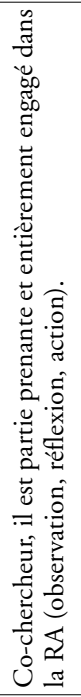 & 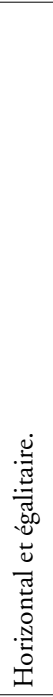 & 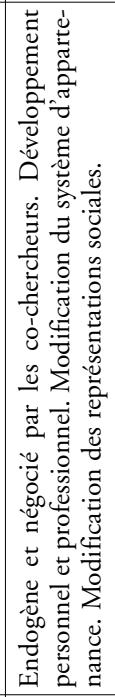 & 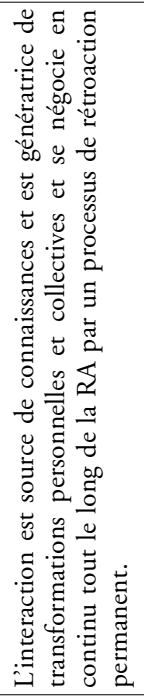 & 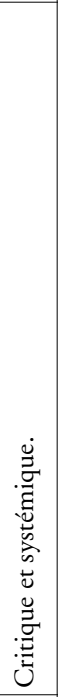 & 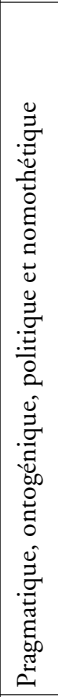 \\
\hline & 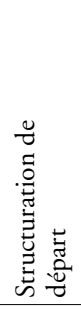 & 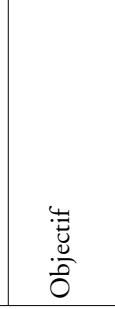 & 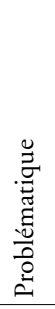 & $\Sigma$ & $\begin{array}{l}\frac{3}{0} \\
\frac{0}{20} \\
\stackrel{2}{\simeq}\end{array}$ & 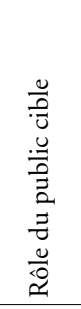 & 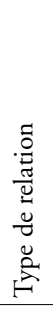 & 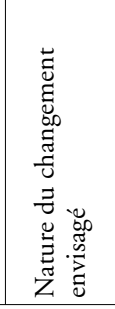 & 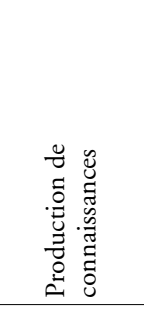 & 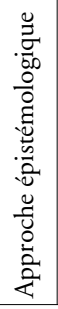 & 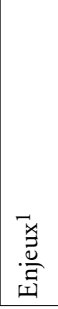 \\
\hline
\end{tabular}


(échanger-interagir), offrir l'excellence, outiller, planifier, réguler, réussir, suivre et unifier» (Mouvet, 2009, pp. 60-70). Ces différents verbes servent la RA tant dans l'assurance d'un relationnel optimum que dans la volonté de son aboutissement. Ces actions permettent à chacun de trouver sa place dans la recherche et valorisent leurs actions et interactions au profit du but envisagé.

Les études empiriques sont également source de principes d'action. Même si les RA sont peu utilisées dans le champ scientifique éducationnel européen et francophone, elles le sont largement dans le monde anglo-saxon. En effet, la pléiade d'études étant telle qu'à la suite de CARN (Collaborative Action Research Network), une revue s'est spécialisée, à partir de 1992, dans ce champ d'investigation («Educational Action Resarch») avec pour objectif de le promouvoir. Nous retrouvons cet attrait dans le champ éducationnel canadien sous le terme essentiellement de recherche collaborative, s'inspirant des préceptes de la RA, notamment avec les études menées entre autres par Desgagné et al. (2001), Tremblay et Bonnelli (2007) et les articles plus théoriques de Savoie-Zajc (2001, 2007). Dans un cadre scolaire, plusieurs études empiriques ont mis en évidence des bénéfices tant pour les enseignants participants que pour les étudiants de tout âge (adaptation et progrès scolaires, pour exemples). Parmi les nombreux auteurs, nous pouvons ainsi citer Vogrinc et Valen i Zuljan (2009) qui ont confirmé expérimentalement la finalité formative de la RA, en tant que processus collaboratif contribuant au développement professionnel. Brighton et Moon (2007) ont, quant à elles, énoncé sept potentialités liées à cette forme de recherche présentées en termes de capacités à:

- résoudre les problèmes;

- faire jouer aux éducateurs un rôle actif au sein de l'école;

- créer une culture scolaire permettant le changement;

- individualiser à partir de collaboration;

- transformer les professeurs en étudiants cherchant à réduire l'écart entre leur pratique et leur vision de l'éducation;

- encourager les enseignants à réfléchir sur leur pratique;

- promouvoir un processus de «testing» d'idées nouvelles.

\section{Pour conclure}

Dans cet article, nous avons voulu présenter l'historique, les définitions, les principes axiologiques de cette recherche et les moyens qui la définissent i.e. finalités, fondements épistémologiques et instrumentations. Le constat qui se fait est que la RA est en perpétuelle évolution et qu'il est vain de vouloir la circonscrire à une définition unique au risque «de la voir réduire à une simple méthodologie» (Dolbec $\&$ Prud'Homme, 2009). Elle possède sans conteste des qualités intrinsèques qui permettent aux acteurs pédagogiques quels qu'ils soient (enseignants, parents d'élèves, pédagogues, éducateurs, etc.) de s'interroger sur leur praxis et l'environ- 
nement dans lequel ils interviennent, de mettre en œuvre des actions qui pourront l'améliorer, de réfléchir sur leur impact afin d'approfondir leurs connaissances et ainsi influer sur le monde réel. Placer l'éducateur au centre de la recherche en tant que co-chercheur et la récursivité des apprentissages en sont ses principes fondateurs favorisant la praxéologie et permettant une appropriation du pouvoir. En suscitant les échanges et en valorisant la prise de conscience des savoir-faire des acteurs tout au long de sa mise en œuvre, elle devient source d'appropriation individuelle et collective de la connaissance des changements nécessaires (Tremblay \& Bonnelli, 2007).

\section{Notes}

1 Selon les termes et définitions empruntés à Van der Maren (2003).

\section{Références}

Anadón, M. \& Savoie-Zajc, L. (2007). La recherche-action dans certains pays anglo-saxons et latino-américains. Une forme de recherche participative. In M. Anadón (Éd.), Recherche participative: Multiples regards (pp. 11-30). Presse Universitaire du Québec.

Bazin, H. (2006). Comparaison entre recherche-action et recherche classique. Bibliographie $R$ - $A$. Consulté le 17 mai 2011 dans http://biblio.recherche-action.fr/document.php?id=137\#tocto2

Berthon, J. P. (2005). La Recherche-Action. «Nouveau millénaire, Défis libertaires». Consulté le 11 juin 2011 dans http://1libertaire.free.fr/Recherche-Action02.html

Bouchard, J. M. (1998). Le partenariat dans une école de type communautaire. In R. Pallascio, L. Julien \& G. Gossemin (Éd.), Le partenariat en éducation. Pour mieux vivre ensemble! (pp. 19-35). Montréal: Editions Nouvelles.

Bouchard, J. M. \& Kalubi, J.C. (2006). Partenariat et recherche de transparence. Des stratégies pour y parvenir. Informations sociales, 133, 50-57.

Brighton, C. M. \& Moon, T. R. (2007). Action Research step-by-step: A tool for educators to change their worlds. Gifted Child Today, 2, 23-27.

Cardinal, P. \& Morin, A. (1993). La modélisation systémique peut-elle se concilier avec la recherche-action intégrale? Educatechnologiques, 1, (2). Consulté le 28 juin 2011 dans http://www.sites.fse.ulaval.ca/reveduc/html/vol1/no2/morin.html

Carr, W. \& Kemmis, S. (1986). Becoming critical: Education, knowledge, and action research. London: Deakin University Press.

Champagne, M. (2007). La pratique de la recherche-action: Entre utopie et nécessité. In H. Dorvil (Éd.), Problèmes sociaux: Tome 3, Théories et méthodologies de la recherche (pp. 463490). St-Nicolas: Presse de l'Université du Québec.

Coenen, H. (2001). Recherche-action: Rapports entre chercheurs et acteurs. Revue internationale de psychosociologie, 16-17, (7), 19-32.

Collier, J. (1945). United States of Indian administration as a laboratory of ethnic relations. Social Research, 12, 265-303.

de Landsheere, G. (1976). Introduction à la recherche en éducation. Liège: Georges Thone.

Desgagné, S., Bednarz, N., Lebuis, P., Poirier L. \& Couture, C. (2001). L'approche collaborative en éducation: Un rapport nouveau à établir entre recherche et formation. Revue des sciences de l'éducation, 27, (1), 33-64.

Deslandes, R. \& Bertrand R. (2005). Motivation of parent involvement in secondary-level schooling. Journal of Educational Research, 98, (3), 164-175. 
Deslandes, R., Potvin, P. \& Leclerc, D. (1999). Family characteristics predictors of school achievement: Parental involvement as a mediator. McGill Journal of Education, 34, (2), 133-151.

Dolbec, A. (2003). La Recherche-Action. In B. Gauthier (Éd.), Recherche sociale: De la problématique à la collecte des données (4e éd.) (pp. 505-540). Presse de l'Université du Québec.

Dolbec, A. \& Prud'Homme (2009). La recherche-action. In B. Gauthier (Éd.), Recherche sociale: De la problématique à la collecte des données (5e éd.) (pp. 531-569). Presse de l'Université du Québec.

Dubost, J. (1988). La question de la spécificité des recherche-action selon les champs. In M. A. Hugon \& C. Seibel (Éd.), Recherches impliquées, recherche action: Le cas de l'éducation (pp. 44-46). Bruxelles: De Boeck.

Epstein, J. L. (1996). Improving school-family-community partnerships in the middle grades. Middle School Journal, 28, (2), 43-48.

Epstein, J. L. (2001). School, family, and community partnerships: Preparing educators and improving schools. Boulder, CO: Westview Press.

Ferrara, M. M. (2009). Broadening the myopic vision of parent involvement. The School Community Journal, 19, (2), 123-142.

Georgiou, S. N. \& Tourva, A. (2007). Parental attributions and parental involvement. Social Psychology of Education, 10, 473-482.

Giust-Desprairies, F. (2001). De la recherche-action à l'intervention psychosociale clinique. Revue internationale de psychosociologie, 16-17, 33-46.

Goyette, G. \& Lessard-Hébert, M. (1987). La recherche-action. Ses fonctions, ses fondements et son instrumentation. Québec: Presses de l'Université du Québec.

Green, C. L., Walker, J. M. T., Hoover-Dempsey, K. V. \& Sandler, H. (2007). Parents' motivations for involvement in children's education: An empirical test of a theoretical model of parental involvement. Journal of Educational Psychology, 99, 532-544.

Hill, N. E. \& Tyson D. F. (2009). Parental involvement in middle school: A meta-analytic assessment of the strategies that promote achievement. Developmental Psychology, 45, (3), 740-763.

Hohlfeld, T. N., Ritzhaupt, A. D. \& Barron, A. E. (2010). Connecting schools, community, and family with ICT: Four-year trends related to school level and SES of public schools in Florida. Computers \& Education, 55, (1), 391-405.

Hoover-Dempsey, K. V., Battiato, A. C., Walker, J. M. T., Reed, R. P., DeJong, J. M. \& Jones, K. P. (2001). Parental involvement in homework. Educational Psychologist, 36, 195-210.

Hoover-Dempsey, K. V. \& Sandler, H. M. (1995). Parental involvement in children's education: Why does it make a difference? Teachers College Record, 95, 310-331.

Hoover-Dempsey, K. V. \& Sandler, H. M. (2005). Final performance report for OERI Grant \# R305T01673. The social context of parental involvement: A path to enhanced achievement. Presented to project monitor, Institute of Education Sciences and U.S Department of Education.

Hoover-Dempsey, K. V., Walker, J. M. T., Jones, K. P. \& Reed, R. P. (2002). Teachers involving parents (TIP): An in-service teacher education program for enhancing parental involvement. Teaching and Teacher Education, 18, 843-867.

Kemmis, S. \& McTaggart, R.. (1988). The Action research planner (3rd ed.). Geelong: Deakin University.

King, J. A. \& Lonnquist, M. P. (1994). A review of writing on action research (1944-present). Communication au Colloque de l'American Educational Research Association. New York.

Kolb, D. A. (1984). Experiental learning: Experience as the source of learning and development. Englewood Cliffs: Prentice Hall.

Lapassade, G. (1993). De l'ethnographie de l'école à la nouvelle recherche-action. Document Dactylographie, Université Paris VIII. Consulté le 14 juin 2011 dans http://biblio.recherche-action.fr 
Mouvet, B. (Éd.). (2009). Construire des réseaux porteurs d'innovations scolaires: Le projet «Solidarité». Les Éditions de l'Université de Liège.

Mouvet, B., Munten, J. \& Jardon, D. (2001, septembre). Vers un référentiel critique des mesures actuelles de prévention et de lutte contre la violence scolaire (Rapport de recherche en éducation $n^{\circ}$ 69/99), non publié, Liège.

Paillé, P. (1994). Pour une méthodologie de la complexité en éducation: Le cas d'une recherche-action-formation. Revue canadienne de l'éducation, 19, (3), 215-230.

Paillé, P. (2007). La méthodologie de recherche dans un contexte de recherche professionnalisante: Douze devis méthodologiques exemplaires. Recherches Qualitatives, 27, (2), 133151.

Perrett, G. (2003). Teacher development through action research. A case study in focused action research. Australian Journal of Teacher Education, 7, (2), 1-10.

Poncelet, D. \& Voz, G. (2004). Une analyse critique de la méthodologie de la recherche-action à la lumière d'une expérience de terrain récente menée au sein du SPE. Cahiers du Service de Pédagogie expérimentale, 17-18, 151-175.

Rouleau, L. (2007). Théories des organisations. Presse de l'Université du Québec.

Savoie-Zajc, L. (2001). La recherche-Action en éducation: Ses cadres épistémologiques, sa pertinence, ses limites. In M. Anadón (2001), Nouvelles dynamiques de Recherche en action (pp. 15-49). Québec: Presses de l'Université Laval.

Schön, D. (1987). The reflective practitioner: How professionals think in action. New York: Basic Books.

Shumsky, A. (1956). Cooperation in Action research: A rationale. Journal of Educational Sociology, n³0. In A.-M. Thirion (1980), Tendances actuelles de la recherche action. Examen critique. Thèse de doctorat, non publiée. Liège: Institut de Psychologie et des Sciences de l'Éducation.

Stenhouse, L. (1975). An introduction to curriculum research and development. London: Heinemann.

Stringer, E.T. (1999). Action Research (2e éd.). Thousand Oaks, CA: Sage.

Taylor, M. (2002). Action research in workplace education. A handbook for literacy instructors. Consulté le 22 août 2011 dans http://www.nald.ca/library/learning/action/cover.htm

Tisseron, S. (2008). Recherche-action. Le jeu de rôle à l'école maternelle: Une prévention de la violence par un accompagnement aux images. Rapport définitif. Consulté le 22 août 2011 dans http:// www.squiggle.be/PDF_Matiere/09_Jeu_de_role_Tisseron.pdf

Tremblay, G. \& Bonnelli, H. (2007). La recherche-action comme moyen pour favoriser la réussite scolaire des garçons aux études collégiales. Education et francophonie, 35, (2), 6277.

Van der Maren, J.-.M. (2003). La recherche appliquée en pédagogie: Des modèles pour l'enseignement (2e éd.). Bruxelles: De Boeck.

Vogrinc, J. \& Valenčič Zuljan, M. (2009). Action research in schools - an important factor in teachers' professional development. Educational Studies, 35, 53-63.

Mots clés: Recherche action, évolution, définition, comparaison, principes d'action 


\section{Handlungsforschung: Kennen, begleiten und bewirken Verän- derungen in den Bildungswissenschaften.}

\section{Zusammenfassung}

Dieser Artikel hat zum Ziel, die Theorie der Handlungsforschung, angewendet auf Bildungswissenschaften, darzustellen. Eine Literaturanalyse ermöglichte die Entwicklung der besagten Theorie seit den 1940er Jahren zu verfolgen und eine, auf epistemologischen Kriterien basierende Definition vorzuschlagen, welche die notwendigen Aktionsgrundsätze besonders hervorhebt. In tabellarischer Form wird die Handlungsforschung der experimentellen Forschung gegenüber gestellt.

Schlagworte: Handlungsforschung, Geschichte, Definition, Vergleich, Aktionsprinzipien

\section{Ricerca-azione: conoscere, accompagnare e provocare il cam- biamento in scienza dell'educazione.}

\section{Riassunto}

Quest'articolo ha per obiettivo la presentazione della teoria della ricerca-azione applicata sulle scienze educative. La rivista della letteratura ci ha permesso di seguire la sua evoluzione dagli anni 1940 e di proponere una definizione basata sui criteri del'epistemologia che la caratterizzano. Di più metteremo in evidenza i suoi principi d'azione. Presentiamo una tabella comparativa che ci permette di distinguere la ricerca-azione della ricerca sperimentale.

Parole chiave: Ricerca azione, evoluzione, definizione, confronto, principi d'azione

\section{Action Research: Knowing, accompanying and causing a change in Education sciences.}

\footnotetext{
Abstract

This article aims to present the theory of action research applied on educational sciences. The review of literature permitted to follow its evolution since the years 1940 , then to define it thanks to it epistemological criteria to highlight it with its acting principles. A table compares action research with experimental research.
}

Key words: Action research-, evolution-, definition-, comparison-, principles of action 